BMJ Open Diabetes Research \& Care

\title{
Trends in prevalence of pre-existing diabetes and perinatal outcomes: a large, population-based study in Catalonia, Spain, 2006-2015
}

\author{
Lucia Gortazar, ${ }^{1,2}$ Albert Goday, ${ }^{1,2,3}$ Juana Antonia Flores-Le Roux (D) ,, 1,2,3 \\ Eugènia Sarsanedas, ${ }^{4}$ Antonio Payà, ${ }^{5}$ Laura Mañé, ${ }^{1,2}$ Juan Pedro-Botet, ${ }^{1,2,3}$ \\ David Benaiges ${ }^{1,2,3,6}$
}

To cite: Gortazar L, Goday A, Flores-Le Roux JA, et al. Trends in prevalence of pre-existing diabetes and perinatal outcomes: a large, population-based study in Catalonia, Spain, 2006-2015. BMJ Open Diab Res Care 2020;8:e001254. doi:10.1136/ bmjdrc-2020-001254

Received 5 February 2020 Revised 17 June 2020 Accepted 13 July 2020

Check for updates

(c) Author(s) (or their employer(s)) 2020. Re-use permitted under CC BY-NC. No commercial re-use. See rights and permissions. Published by BMJ.

${ }^{1}$ Department of Endocrinology and Nutrition, Hospital del Mar, Barcelona, Spain

${ }^{2}$ Department of Medicine, Universitat Autònoma de Barcelona, Barcelona, Spain ${ }^{3}$ Institut Hospital del Mar d'Investigacions Mèdiques (IMIM), Barcelona, Spain ${ }^{4}$ Health Information Management Department, Hospital del Mar, Barcelona, Spain

${ }^{5}$ Department of Gynaecology and Obstetrics, Hospital del Mar, Barcelona, Spain ${ }^{6}$ Consorci Sanitari de l'Alt Penedès Garraf, Vilafranca del Penedès, Spain

Correspondence to Dr Juana Antonia Flores-Le Roux;

94066@parcdesalutmar.cat

\section{ABSTRACT}

Introduction This study aims to assess trends in the prevalence of pre-existing diabetes and whether the risk of adverse perinatal outcomes decreased in women between 2006 and 2015 in Catalonia, Spain.

Research design and methods A population-based study of 743762 singleton deliveries between 2006 and 2015 in Catalonia, Spain, was conducted using data from the Spanish Minimum Basic Data Set. Cases of type 1 diabetes (T1DM) and 'type 2 diabetes and other pre-existing diabetes' ('T2DM and other PGD') were identified using International Classification of Diseases, Ninth Revision, Clinical Modification codes. Crude and age-adjusted annual prevalences were calculated. Poisson regression model was used to assess trends in prevalence and perinatal outcomes during the study period.

Results Overall prevalences of pre-existing diabetes, T1DM and 'T2DM and other PGD' were 0.52\% (95\% $\mathrm{Cl} 0.51$ to 0.54$), 0.17 \%$ (95\% Cl 0.17 to 0.18 ) and $0.35 \%$ (95\% Cl 0.33 to 0.36$)$, respectively. From 2006 to 2015 , rates increased for pre-existing diabetes (from $0.43(95 \% \mathrm{Cl} 0.39$ to 0.48$)$ to $0.56 \%$ (0.50 to $0.62), p<0.001), T 1 D M$ (from $0.14(0.11$ to 0.17$)$ to $0.20 \%$ (0.17 to 0.23$), p<0.001)$ and 'T2DM and other PGD' (from 0.29 (0.25 to 0.33 ) to $0.36 \%$ (0.31 to $0.40), p<0.001)$. Pre-eclampsia rose in women with pre-existing diabetes (from $4.38 \%$ to $8.97 \%$, adjusted $\mathrm{p}<0.001$ ), T1DM (from $3.85 \%$ to $12.88 \%, \mathrm{p}=0.005$ ) and 'T2DM and other PGD' (from $4.63 \%$ to $6.78 \%$, adjusted $p=0.01$ ). Prevalence of prematurity, cesarean section and small for gestational age remained stable in all diabetes groups. However, the prevalence of macrosomia fell in women with pre-existing diabetes (from $18.18 \%$ to $11.9 \%$, adjusted $\mathrm{p}=0.011$ ) and 'T2DM and other PGD' (from $14.71 \%$ to $11.06 \%$, non-adjusted $p=0.022$, adjusted $p=0.305$ ) and large for gestational age decreased in all diabetes groups (from $39.73 \%$ to $30.25 \%$ in pre-existing diabetes, adjusted $p=0.004$ ). Conclusions The prevalence of pre-existing diabetes increased significantly in Catalonia between 2006 and 2015. Despite improvements in outcomes related to excessive birth weight, pre-eclampsia rates are rising and overall perinatal outcomes in women with preexisting diabetes continue to be markedly worse than in the population without diabetes.

\section{Significance of this study}

What is already known about this subject?

- The prevalence of pre-existing diabetes in pregnancy is increasing in Europe and worldwide.

- Women with pre-existing diabetes have poorer perinatal outcomes than women without diabetes.

- Few studies have evaluated trends in perinatal outcomes in women with pre-existing diabetes with different findings.

What are the new findings?

- The prevalence of pre-existing diabetes (type $1 \mathrm{di}-$ abetes and 'type 2 diabetes and other pre-existing diabetes') has steadily increased between 2006 and 2015 in Catalonia.

- The risk of adverse perinatal outcomes in women with pre-existing diabetes remains significantly high compared with women without diabetes.

- An increasing trend in pre-eclampsia, a stable trend in cesarean rates, prematurity and small for gestational age, and a downward trend in macrosomia and large for gestational age have been detected in women with pre-existing diabetes.

\section{How might these results change the focus of} research or clinical practice?

- As pre-existing diabetes in pregnancy becomes more common, there is an urgent need for prevention strategies, early diagnosis and improvement in maternal clinical care in order to improve perinatal outcomes.

\section{INTRODUCTION}

Diabetes among women of childbearing age is a growing concern worldwide ${ }^{1-5}$ and carries an increased risk of pregnancy complications and adverse perinatal outcomes. ${ }^{16-8}$ In the St Vincent Declaration in 1989, the WHO and the International Diabetes Federation declared, as a 5-year goal, that 'the outcome of diabetic pregnancy should approximate that of the non-diabetic pregnancy'. 
Preconception counseling with optimization of glycemic control and appropriate management of associated comorbidities has proved to lower adverse perinatal outcome rates in women with pre-existing diabetes. ${ }^{9} 10$ However, despite promising improvements in specialized units, population-based studies reported that women with pre-existing diabetes continue to have poorer outcomes than those without diabetes and trends in perinatal outcomes are not improving. ${ }^{146711}$ No population-based data from Southern European countries are currently available. Thus, the aim of the present study was to assess trends in the prevalence of pre-existing diabetes mellitus in pregnancy between 2006 and 2015 in Catalonia, Spain, and whether adverse perinatal outcomes in these women improved over the study period.

\section{PATIENTS AND METHODS}

Hospital delivery discharges were collected from the Minimum Basic Data Set for Hospital Discharge (CMBD$\mathrm{AH}$ ), which is a database that includes all hospital admission reports from both public and private hospitals in Catalonia, the second largest autonomous community in Spain in terms of population ( 7.5 million inhabitants). All hospital discharge reports for singleton births in women aged 15-45 years with diagnostic-related group codes 370-375 (cesarean and vaginal deliveries) from January 2006 to December 2015 were analyzed. Diabetes cases were identified for each hospital delivery discharge report using International Classification of Diseases, Ninth Revision, Clinical Modificacion (ICD-9-CM) codes. Patients diagnosed with 'gestational diabetes' (GDM) (codes 648.8x) were excluded. Type 1 diabetes (T1DM) was identified by ICD-9-CM codes 250.x1 or 250 . $\mathrm{x} 3$. The 'type 2 and other pre-existing diabetes' group ('T2DM and other PGD') was defined as women with pre-existing diabetes who did not have ICD-9-CM codes for T1DM, and included women with type 2 diabetes (T2DM) diagnosis codes 250.x0 or 250.x2) and 'diabetes mellitus, pre-pregnancy' codes 648.0x) listed anywhere on their discharge report. The overall pre-existing diabetes group comprised all women with diabetes prior to pregnancy and included the T1DM and 'T2DM and other PGD' groups. Data on maternal characteristics included age, hypertension (ICD-9 codes 642.0-642.2 or 401-405), dyslipidemia (ICD-9 code 272), and smoking status (ICD-9 code 649.0 or 305.1). Regarding obstetric complications, pre-eclampsia was defined as ICD-9 codes 642.4-642.6 and cesarean section by ICD-9 codes 74.0, $74.4,74.9,74.91,74.99,669.7$, and 669.71 listed anywhere on the discharge report.

Macrosomia was defined according to the American College of Obstetricians and Gynecologists (ACOG) as birth weight $\geq 4000 \mathrm{~g}$. Preterm delivery included birth before 37 weeks' gestation according to the WHO and the ACOG. Term deliveries were categorized according to the ACOG as early term (37 $0 / 7$ weeks of gestation through $386 / 7$ weeks of gestation), full term (39 0/7 weeks of gestation through $406 / 7$ weeks of gestation), late term $(410 / 7$ weeks of gestation through $416 / 7$ weeks of gestation), and post-term (42 $0 / 7$ weeks of gestation and beyond). ${ }^{12}$ The birthweight percentiles for gestational age (30-44 weeks) were established using non-customized percentiles, with data obtained from the birth weight by the gestational age population reference curves of Catalonia. A newborn was considered to be large for gestational age (LGA) if the birth weight was above the estimated 90th percentile for the baby's sex and gestational age and small for gestational age (SGA) if the birth weight was below the 10th percentile.

Data from all delivery reports were anonymized.

\section{Statistical analysis}

Prevalence was calculated by dividing the cases of preexisting diabetes, T1DM and 'T2DM and other PGD' by the total number of deliveries during each period. Crude and age-adjusted annual prevalences were calculated using direct standardization to the maternal age structure of the whole study population. Maternal characteristics and perinatal outcomes of women with diabetes were compared using multivariate logistic regression models, adjusted for maternal age and smoking status. Time trends in crude and adjusted prevalences of diabetes and perinatal outcomes were estimated using a Poisson regression model. All statistical analyses were two-tailed, with $\mathrm{p}<0.05$ considered significant. All analyses were made with the statistical software package IBM SPSS Statistics V.25.0.

\section{RESULTS}

From January 2006 to December 2015, 743762 hospital deliveries were recorded in Catalonia. Data on global trends in annual deliveries, mean maternal age in the population of individuals without diabetes, prevalence of GDM, and trends in perinatal outcomes in women with GDM and women without diabetes have been published elsewhere. $^{13}$

Of all hospital deliveries, 3882 were identified as having pre-existing diabetes mellitus $(0.52 \%, 95 \%$ CI 0.51 to $0.54)$. Of these, $1299(33.5 \%)$ were classified as T1DM and $2583(66.5 \%)$ as 'T2DM and other PGD'. As shown in table 1 , women with pre-existing diabetes were older and had higher hypertension and dyslipidemia rates than those without diabetes. Women with 'T2DM and other PGD' were older than those with T1DM. No differences in the prevalence of hypertension or dyslipidemia were observed between women with 'T2DM and other PGD' and T1DM; however, smoking was more frequent in women with T1DM.

Trends in crude and age-adjusted prevalences of preexisting diabetes in pregnancy, T1DM and 'T2DM and other PGD' are shown in figure 1. Rates of pre-existing diabetes rose from $0.43 \%$ (95\% CI 0.39 to 0.48 ) in 2006 to $0.56 \%$ ( $95 \%$ CI 0.50 to 0.62 ) in 2015 . The prevalence of T1DM increased from $0.14 \%$ (95\% CI 0.11 to 0.17 ) 


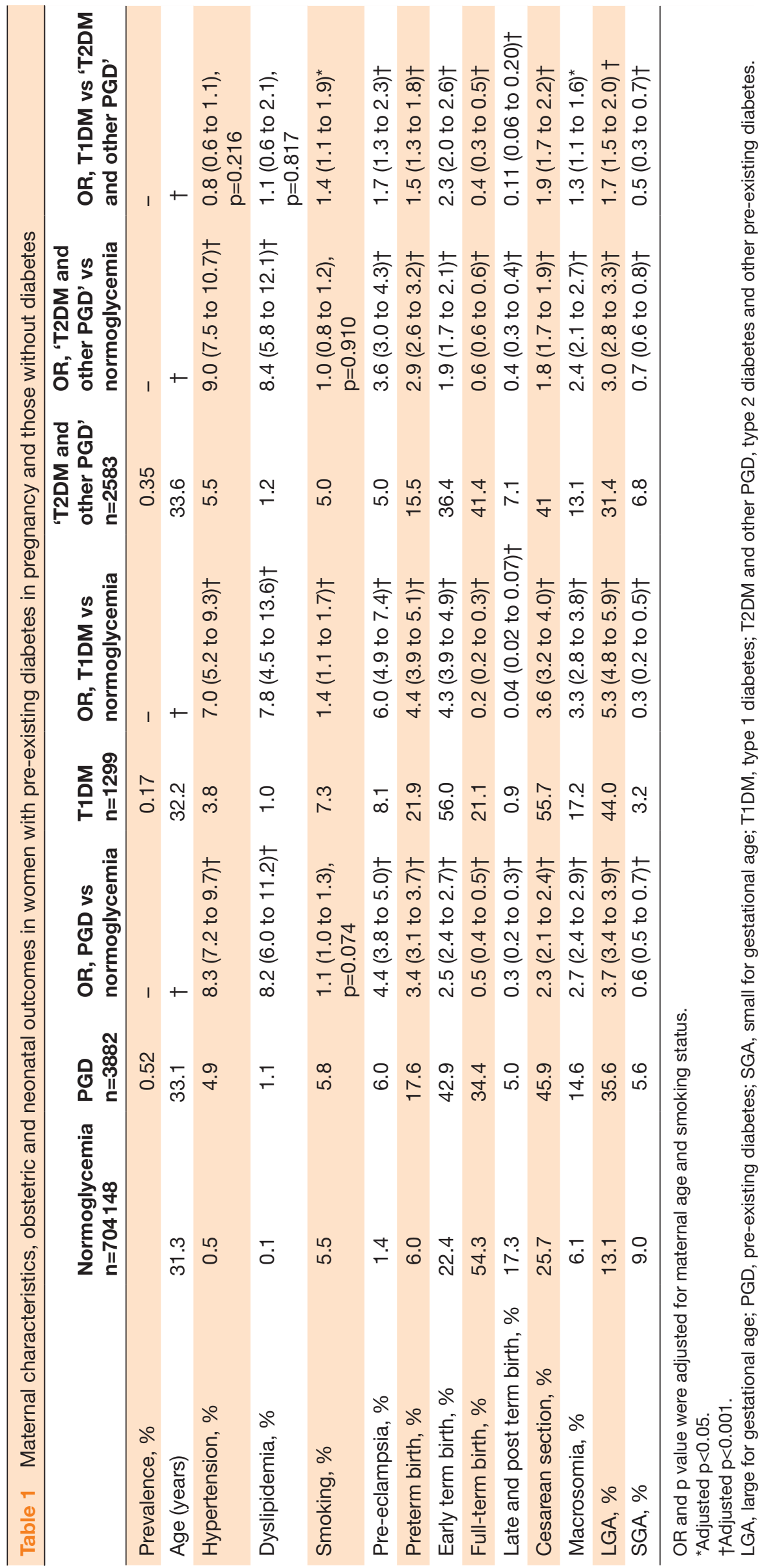




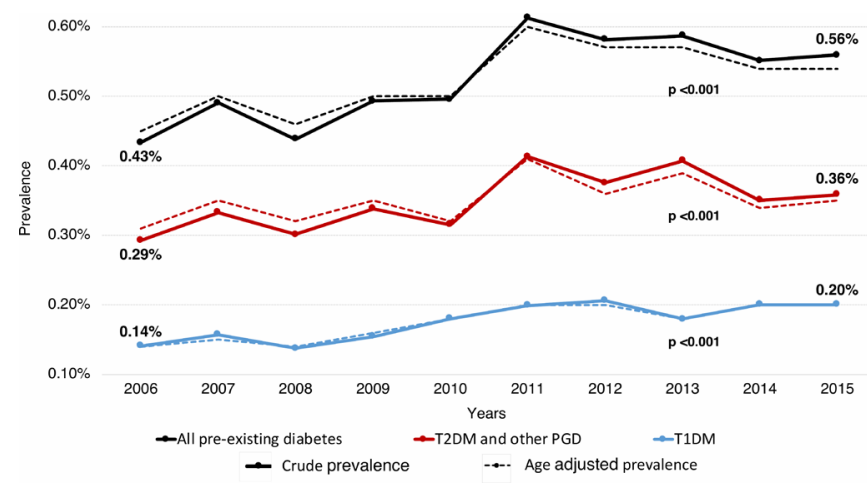

Figure 1 Trends in prevalence of pre-existing diabetes in pregnancy between 2006 and 2015 in Catalonia. P value was adjusted for maternal age. Note that the y axis is truncated. T1DM, type 1 diabetes; T2DM and other PGD, type 2 diabetes and other pre-existing diabetes.

to $0.20 \%$ (95\% CI 0.17 to 0.23 ) and the prevalence of 'T2DM and other PGD' from $0.29 \%$ (95\% CI 0.25 to 0.33 ) to $0.36 \%$ ( $95 \%$ CI 0.31 to 0.40 ) during the study period.
Similarly, increases in age-adjusted trends were observed in women with pre-existing diabetes (from $0.45 \%(95 \%$ CI 0.40 to 0.50 ) to $0.54 \%$ (95\% CI 0.49 to 0.60$)$ ), T1DM (from $0.14 \%$ (95\% CI 0.11 to 0.17 ) to $0.20 \%$ (95\% CI 0.16 to 0.23 ) ) and 'T2DM and other PGD' (from $0.31 \%$ ( $95 \%$ CI 0.27 to 0.35 ) to $0.35 \%$ (95\% CI 0.30 to 0.39$)$ ).

Regarding perinatal outcomes, women with pre-existing diabetes had higher rates of adverse perinatal outcomes, except for SGA, than women without diabetes (table 1). Similarly, with the exception of SGA, the risks for all adverse perinatal outcomes considered were significantly greater in women with T1DM compared with those with 'T2DM and other PGD'. Trends in pre-eclampsia, prematurity, term deliveries, cesarean deliveries, macrosomia, LGA and SGA are shown figures 2 and 3. A growing trend in crude and adjusted rates of pre-eclampsia was observed in women with pre-existing diabetes, T1DM and 'T2DM and other PGD' between 2006 and 2015 (from $4.38 \%$ to $8.96 \%$ in pre-existing diabetes, from $3.85 \%$ to $12.88 \%$ in $\mathrm{T} 1 \mathrm{DM}$, and from $4.63 \%$ to $6.78 \%$ in 'T2DM
A

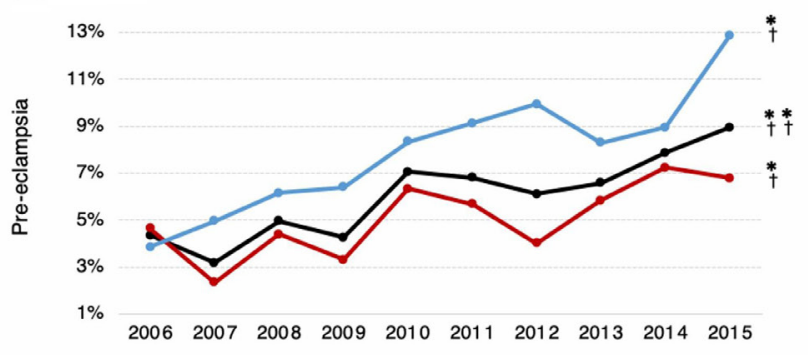

C

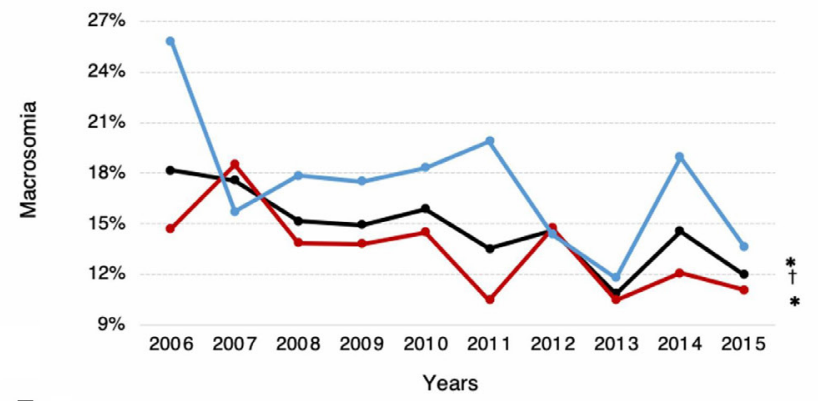

$\mathbf{E}$

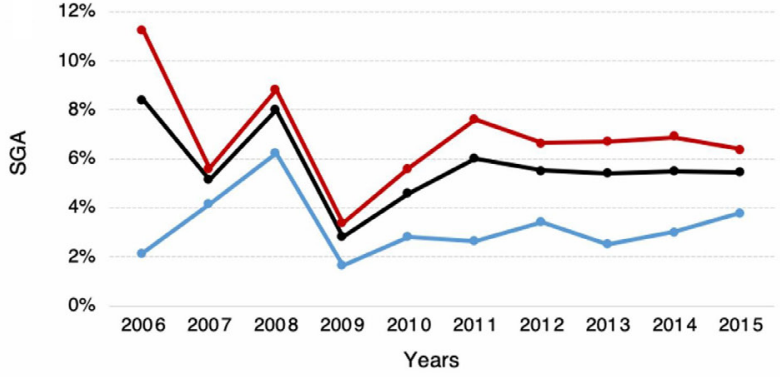

B

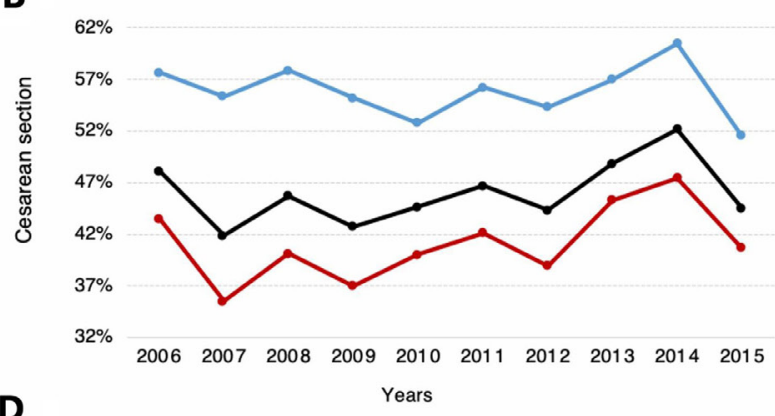

D

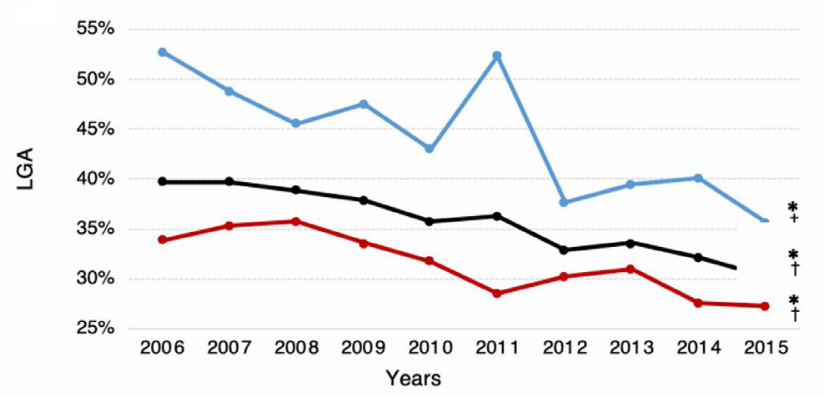

-All pre-existing diabetes -T2DM and other PGD -T1DM

Figure 2 Trends in perinatal outcomes in women with pre-existing diabetes in pregnancy between 2006 and 2015. (A) Preeclampsia, (B) cesarean section, (C) macrosomia, (D) LGA and (E) SGA; ${ }^{*} p<0.05,{ }^{* *} p<0.001,{ }^{\dagger}$ adjusted $p<0.05,{ }^{+\dagger}$ adjusted $p<0.001$. Note that in $A-E$, the $y$ axis is truncated. LGA, large for gestational age; SGA, small for gestational age; T1DM, type 1 diabetes; T2DM and other PGD, type 2 diabetes and other pre-existing diabetes. 


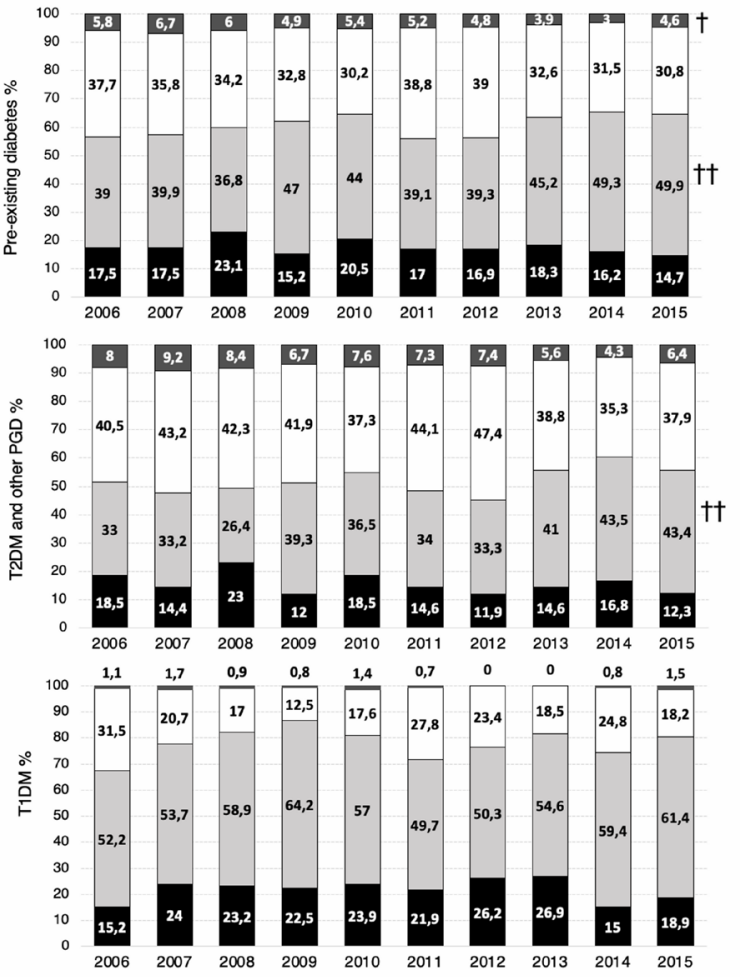

- Preterm birth Early term birth $\quad$ Full term birth $\quad$ - Late and post term birth

Figure 3 Trends in preterm and term deliveries in women with pre-existing diabetes in pregnancy between 2006 and 2015. †Adjusted $p<0.05$, ††adjusted $p<0.001$. T1DM, type 1 diabetes; T2DM and other PGD, type 2 diabetes and other pre-existing diabetes.

and other PGD'). Prematurity and cesarean section rates remained stable for pre-existing diabetes, T1DM and 'T2DM and other PGD' during the study period. However, when analyzing trends on term deliveries, we observed an increasing trend in 'early term birth' in preexisting diabetes and in 'T2DM and other PGD', whereas 'late and post term birth' decreased in women with preexisting diabetes (figure 3).

With respect to fetal growth outcomes, macrosomia rates showed a diminishing trend in women with preexisting diabetes (from $18.2 \%$ to $12 \%$, adjusted $\mathrm{p}=0.011$ ) and those with 'T2DM and other PGD' (from $14.7 \%$ to $11.1 \%$, non-adjusted $\mathrm{p}=0.022$ ); however, the decrease was not statistically significant in the latter group when adjusted for age and smoking status (adjusted $\mathrm{p}=0.305$ ). The prevalence of macrosomia remained stable during the study period in women with T1DM (adjusted $\mathrm{p}=0.119$ ) (figure 2C). LGA rates dropped significantly in women with pre-existing diabetes (from 39.7\% to $30.2 \%$, adjusted $\mathrm{p}=0.004$ ), T1DM (from $52.7 \%$ to $35.6 \%$, adjusted $\mathrm{p}=0.025$ ) and 'T2DM and other PGD' (from $33.8 \%$ to $27.2 \%$, adjusted $\mathrm{p}=0.033$ ) between 2006 and 2015 (figure 2D). Finally, the prevalence of SGA remained stable in all diabetes groups during the study period (figure 2E).

\section{DISCUSSION}

This large, population-based study showed that the prevalence of overall pre-existing diabetes in pregnancy rose steadily in Catalonia, Spain between 2006 and 2015, with a $30 \%$ overall increase. A more pronounced increase was observed in women with T1DM $(43 \%)$, whereas the prevalence of 'T2DM and other PGD' showed a $24 \%$ rise. The frequency of adverse perinatal outcomes in women with pre-existing diabetes remained substantially high compared with women without diabetes. Regarding trends in perinatal outcomes, an increasing trend in preeclampsia, a stable trend in cesarean rates, prematurity and SGA, and a downward trend in macrosomia and LGA in women with pre-existing diabetes were noted.

The overall prevalence of pre-existing diabetes was $0.52 \%$, consistent with that described in other European population-based studies. ${ }^{161114}$ However, studies conducted in the USA and Canada showed higher prevalences of pre-existing diabetes, ranging from $0.8 \%$ to $1.3 \% .^{235}$ Conversely, the only population-based study in Spain analyzed data from 2001 to 2008 and found a much lower prevalence of pre-existing diabetes, at $0.2 \% .^{15} \mathrm{In}$ that study, the same CMBD database and ICD-9-CM classification were used as in the present study. However, the diagnosis of pre-existing diabetes was established using 250.xx codes, whereas 648.0x codes were not included. In our registry, 1688 women were found to have $648.0 \mathrm{x}$ code ('diabetes mellitus pre-pregnancy, excluding GDM'), with none of these women having GDM or T1DM ICD-9-CM codes. These 1688 cases represented almost half $(43 \%)$ the total pre-existing diabetes cases and were therefore considered unrealistic to be excluded. If we had ignored those diagnostic codes, the prevalence would have been $0.29 \%$, certainly underestimating the real figure of pre-existing diabetes. Previous populationbased studies similarly included 648.0x ICD-9-CM codes in their analysis of pre-existing diabetes prevalence. Bardenheier et $a l^{16}$ evaluated the prevalence of preexisting diabetes by considering both 250.xx and 648.0x ICD-9-CM codes and found an $0.8 \%$ prevalence in 19 US states between 2000 and 2010. Moreover, Albrecht et $a l^{17}$ reported a $0.16 \%$ prevalence of 'unspecified diabetes', which included women with ICD-9-CM codes 648.0x, and an overall prevalence of pre-existing diabetes of $0.66 \%$. The 'unspecified diabetes' group accounted for $24 \%$ of all pre-existing diabetes cases, thus largely contributing to the described figure of pre-existing diabetes. ${ }^{17}$ Conversely, other studies conducted in the USA using ICD-9-CM codes analyzed only 250.xx codes, excluding 648.0x ; however, those studies also identified diabetes cases through insulin prescription and glycated hemoglobin levels to avoid underestimation of the prevalence. The observed prevalences were $0.8 \%$ from 1996 to 2014 in Northern California ${ }^{5}$ and 1.3\% from 1995 to 2005 in Southern California. ${ }^{3}$

The growing trend in the number of pregnancies complicated by pre-existing diabetes was also consistent with other studies in Europe ${ }^{16141518}$ and the USA. ${ }^{31617}$ 
The rise in diabetes prevalence during pregnancy reflects background trends in T2DM in the general population and in particular in young adults and adolescents. ${ }^{19}{ }^{20} \mathrm{In}$ this regard, a female preponderance has been described in young-onset T2DM. ${ }^{19}$ Obesity, inadequate diet, lack of physical activity, socioeconomic factors, changes in migration patterns and increasing maternal age might be some of the key factors involved in the rise of T2DM during pregnancy. Moreover, as screening for unrecognized preexisting diabetes at the first antenatal visit is encouraged by various societies, ${ }^{21}{ }^{22}$ a greater number of T2DM cases might be diagnosed. For many years, GDM was defined as any degree of glucose intolerance with onset or first recognition during pregnancy. ${ }^{21}$ Nevertheless, as the prevalence of undiagnosed T2DM cases in women of childbearing age continued to increase, the term 'overt diabetes' was coined by the International Association of Diabetes and Pregnancy Study Groups (IADPSG) in 2010 as pregnant women who meet the criteria for diabetes in the non-pregnant state but were not previously diagnosed with diabetes. ${ }^{23}$ Therefore, since 2010, the American Diabetes Association (ADA) has endorsed testing for overt diabetes $^{24}$ or pre-existing pregestational diabetes ${ }^{21}$ at the first antenatal visit in women at high risk for T2DM, and since 2013 the Endocrine Society has recommended universal first-trimester screening for overt diabetes. ${ }^{22}$ As the nomenclature and diagnostic criteria of overt diabetes changed over the study period, and given there is no specific ICD-9-CM code for overt diabetes, we could speculate that some of these overt diabetes cases might have been identified with $648.0 *$ code ('diabetes mellitus, pre-pregnancy').

The increasing prevalence of T1DM in pregnant women found in the present study is in line with previous reports on a worldwide rising prevalence of T1DM which remains unexplained. ${ }^{202526}$ We could also speculate that improvements in prepregnancy counseling, with optimization of glycemic control and appropriate management of related comorbidities, as well as greater access to assisted reproductive technology might have helped women with diabetes to conceive and ultimately give birth to a live infant, thereby increasing the rates of both T1DM and T2DM in pregnancy.

The present study showed that women with pre-existing diabetes had higher rates of cardiovascular risk factors and worse perinatal outcomes than women without diabetes.

In particular, women with T1DM had an increased risk of pre-eclampsia, prematurity, cesarean section, macrosomia and LGA than women with 'T2DM and other PGD'. These findings concur with those of some previous population-based studies. ${ }^{6727}$ However, the meta-analysis of Balsells $e t a l^{28}$ showed a higher risk of cesarean deliveries in women with T1DM but no statistically significant differences in pre-eclampsia, macrosomia and LGA compared with T2DM.

Rates of cesarean deliveries were $55.7 \%$ for women with T1DM and $41 \%$ for 'T2DM and other PGD' (compared with $25.7 \%$ in women with normoglycemia). Although these rates are similar to those reported in other populations, ${ }^{6729}$ a twofold increase in the risk of cesarean section in women with pre-existing diabetes remains high. In this respect, recommendations for cesarean section in women with diabetes in Spain are no different from those for women with normoglycemia, except for estimated birth weight $>4500 \mathrm{~kg}$ or prior shoulder dystocia. ${ }^{30}$ According to the Spanish Group of Diabetes and Pregnancy (GEDE), induced labor might be considered for women with diabetes from 38 weeks of gestation, and from 37 weeks if adequate obstetric and glycemic control cannot be assured. In this context, we could speculate that labor induction might increase the risk of cesarean section. Previous evidence on the effect of induction of labor on cesarean section rates (compared with expectant management) has provided conflicting results. Results from observational studies have generally reported an increase in the rate of cesarean section, ${ }^{31}{ }^{32}$ whereas evidence from randomized controlled trials and metaanalysis shows no difference or a reduction in risk. ${ }^{33-35}$ Taking this into consideration, and given that increased birth weight does not fully explain the increased risk of cesarean section in women with diabetes during pregnancy, other factors such as practice patterns or physician referrals to high-risk care have been suggested to contribute to high rates of cesarean delivery in these women. ${ }^{36}$

One of the most alarming results regarding trends in adverse perinatal outcomes was the rise in pre-eclampsia rates in women from all diabetes groups. In this respect, Bardenheier et $a l^{16}$ found that the proportion of preexisting diabetes in deliveries complicated with preeclampsia significantly increased from 2000 to 2010. This rise was also noticed in our cohort of pregnant women without diabetes ${ }^{13}$ and in other previous studies conducted in women with normoglycemia, ${ }^{37} 38$ and may account for population-level changes in prepregnancy body mass index (BMI), parity, smoking and pre-existing maternal conditions. ${ }^{39} 40$

Although no drop in cesarean deliveries and prematurity rates in women with pre-existing diabetes was observed, it must be pointed out that these outcomes increased in women without diabetes in our cohort over the study period. ${ }^{13}$ Stable rates were also observed in women with pre-existing diabetes by Bell et $a l^{11}$ in the North of England between 1996 and 2004 and by Khalifeh $e t a l^{40}$ in Dublin between 1999 and 2008. However, mixed results were found in other surveys analyzing trends in prematurity and cesarean deliveries in women with pre-existing diabetes. ${ }^{1616}$

Regarding trends in birthweight outcomes, we detected a falling trend for LGA in all diabetes groups and for macrosomia in the overall pre-existing diabetes group. Considering that outcomes related to excessive fetal growth are the most likely to be influenced by glycemic control, the decreasing rates of LGA/macrosomia might suggest a certain degree of improvement in metabolic 
control and diabetic care in women with pre-existing diabetes. Moreover, this downward trend contrasts with the increasing rate of LGA observed in women without diabetes in our database. Varied evidence has been published in this respect. ${ }^{16}$

With reference to diabetes care in pregnancy in Catalonia, management is based on recommendations of the GEDE, which is composed of members of the Spanish Society of Obstetrics and Gynecology, the Spanish Paediatrics Association, and the Spanish Diabetes Society. The GEDE's management guidelines and recommendations are common to all pre-existing diabetes (including T1DM and T2DM). ${ }^{41}$ Nevertheless, previous evidence has shown that women with T2DM have lower rates of prepregnancy care than women with T1DM. ${ }^{27}{ }^{28}$ Furthermore, greater use of technology and the complexity of insulin adjustment in women with T1DM might lead to a closer follow-up during pregnancy. Although clinical guidelines and recommendations made by the GEDE are widely followed across maternity hospitals, a study published in 2015 by this group revealed an unequal access to preconception clinic, to new therapies such as continuous subcutaneous insulin infusion and to nurse support among maternity hospitals in Spain. ${ }^{42}$ The GEDE's clinical guidelines were revised in $2006^{41}$ and subsequently updated in $2015 .^{30}$ Therefore, no major changes in management recommendations of diabetes in pregnancy were made during the study period. However, a generalization in the use of insulin analogs and the increasing access to technology (in particular in women with T1DM) were observed in clinical practice over the study period. Furthermore, as a result of ADA, IADPSG and WHO's 2010 recommendations to test for overt diabetes, more women might have been diagnosed of pre-existing diabetes in early pregnancy in the second half of the study period, therefore affecting the subsequent management and care of these patients.

An increasing trend of pre-existing diabetes in pregnancy was found in our study, and as the incidence of diabetes continues to rise in the general population, especially in young age groups, this upward trend is unfortunately expected to continue. Furthermore, despite advances in clinical care, overall results show that the goals stated in the St Vincent Declaration are far from being achieved. Increasing efforts are needed to curb the upward trend of T2DM prevalence and reduce the rates of adverse perinatal outcomes in women with pre-existing diabetes. In this respect, diabetes prevention and early identification of unknown diabetes in women of childbearing age are crucial. Efforts should be made to improve both preconception and perinatal healthcare by ensuring glycemic optimization and correct assessment of maternal comorbidities.

The main strength of the present study lies in the population-based data on $>700000$ deliveries over a 10-year period, providing valuable epidemiological evidence. Moreover, to our knowledge, this is the only recent study to analyze trends in pre-existing diabetes in
Southern Europe. The CMBD database covers all hospital admissions in Catalonia and the ICD-9-CM remained the coding system from 1981 to the end of our study period. Furthermore, we were not only able to analyze the epidemiology of pre-existing diabetes overall, but also to identify the type of pre-existing diabetes using specific ICD-9-CM codes.

Some limitations must also be considered. Our results are based on the retrospective analysis of an administrative database and diagnoses were established according to ICD-9-CM codes, without knowledge of the criteria used to make the diagnoses; therefore, issues related to the validity and reliability of coding may arise. Codification of diabetes subtypes and perinatal outcomes might, in some cases, have been unreliable and the ICD-9-CM codes could not be crosschecked with other clinical databases. Unfortunately, we were unable to obtain data on major perinatal outcomes such as stillbirth, neonatal and perinatal mortality, congenital anomalies, and newborn neonatal intensive care unit admission since linked maternal and neonatal data were not available. Furthermore, data on maternal weight, BMI and ethnicity, and all relevant conditions that may affect pregnancy outcomes were not recorded.

In conclusion, the number of women with pre-existing diabetes in pregnancy, both T1DM and 'T2DM and other PGD', has increased in Catalonia over the last 10 years. Although some improvements in macrosomia and LGA rates were detected in these women, their overall risk for adverse perinatal outcomes remained significantly high compared with non-diabetic pregnancies. The results highlight the need for effective diabetes prevention and control strategies for women of childbearing age which may help protect their health and that of their newborns.

Acknowledgements We thank Miss Christine 0'Hara for review of the English version of the manuscript, Montse Clèries from Unitat d'Informació i Coneixement, Servei Català de la Salut (CatSalut), Marta Albacar from Divisió d'anàlisi de la demanda i I'activitat, Servei Català de la Salut (CatSalut), and Conxa Castell from Servei de Promoció de Vida Saludable i Prevenció de Malalties Cròniques no Transmissibles, Agència de Salut Pública de Catalunya, Departament de Salut, Generalitat de Catalunya.

Contributors All authors made substantial contributions to conception and design, acquisition of data, or analysis and interpretation of data; drafted the article or revised it critically for important intellectual content; gave final approval of the version to be published; and agreed to be accountable for all aspects of the work in ensuring that questions related to the accuracy or integrity of any part of the work are appropriately investigated and resolved. Study concept and design: AG, DB, ES, JAF-LR and LG. Acquisition of data: AG and ES. Analysis and interpretation of data: $A G, D B, E S, J A F-L R$ and LG. Drafting of the manuscript: LG. Critical revision of the manuscript for important intellectual content: AG, AP, DB, ES, JAF-LR, JP-B, LG and LM. Statistical analysis: DB, ES and LG.

Funding The authors have not declared a specific grant for this research from any funding agency in the public, commercial or not-for-profit sectors.

\section{Competing interests None declared.}

Patient consent for publication Not required.

Ethics approval The study was conducted according to the principles of the Declaration of Helsinki and approved by the clinical research ethics committee of our institution (CEIC-Parc de Salut MAR, number 2017/7209/I).

Provenance and peer review Not commissioned; externally peer reviewed. 
Data availability statement Data are available upon request from the authors.

Open access This is an open access article distributed in accordance with the Creative Commons Attribution Non Commercial (CC BY-NC 4.0) license, which permits others to distribute, remix, adapt, build upon this work non-commercially, and license their derivative works on different terms, provided the original work is properly cited, appropriate credit is given, any changes made indicated, and the use is non-commercial. See: http://creativecommons.org/licenses/by-nc/4.0/.

ORCID iD

Juana Antonia Flores-Le Roux http://orcid.org/0000-0002-3760-5017

\section{REFERENCES}

1 Beyerlein A, von Kries R, Hummel M, et al. Improvement in pregnancy-related outcomes in the offspring of diabetic mothers in Bavaria, Germany, during 1987-2007. Diabet Med 2010;27:1379-84.

2 Feig DS, Hwee J, Shah BR, et al. Trends in incidence of diabetes in pregnancy and serious perinatal outcomes: a large, populationbased study in Ontario, Canada, 1996-2010. Diabetes Care 2014;37:1590-6.

3 Lawrence JM, Contreras R, Chen W, et al. Trends in the prevalence of preexisting diabetes and gestational diabetes mellitus among a racially/ethnically diverse population of pregnant women, 19992005. Diabetes Care 2008;31:899-904.

4 Abouzeid M, Versace VL, Janus ED, et al. A population-based observational study of diabetes during pregnancy in Victoria, Australia, 1999-2008. BMJ Open 2014:4:e005394.

5 Peng TY, Ehrlich SF, Crites Y, et al. Trends and racial and ethnic disparities in the prevalence of pregestational type 1 and type 2 diabetes in Northern California: 1996-2014. Am J Obstet Gynecol 2017;216:177.e1-8.

6 Mackin ST, Nelson SM, Kerssens JJ, et al. Diabetes and pregnancy: national trends over a 15 year period. Diabetologia 2018;61:1081-8.

7 Metcalfe A, Sabr Y, Hutcheon JA, et al. Trends in obstetric intervention and pregnancy outcomes of Canadian women with diabetes in pregnancy from 2004 to 2015 . $J$ Endocr Soc 2017;1:1540-9.

8 Lapolla A, Dalfrà MG, Di Cianni G, et al. A multicenter Italian study on pregnancy outcome in women with diabetes. Nutr Metab Cardiovasc Dis 2008;18:291-7.

9 Willhoite MB, Bennert HW, Palomaki GE, et al. The impact of preconception counseling on pregnancy outcomes. The experience of the Maine diabetes in pregnancy program. Diabetes Care 1993;16:450-5.

10 The Diabetes Control Complications Trial Research Group. Pregnancy outcomes in the diabetes control and complications trial. Am J Obstet Gynecol 1996;174:1343-53.

11 Bell R, Bailey K, Cresswell T, et al. Trends in prevalence and outcomes of pregnancy in women with pre-existing type I and type II diabetes. BJOG 2008; 115:445-52.

12 ACOG Committee opinion no 579: definition of term pregnancy. Obstet Gynecol 2013;122:1139-40.

13 Gortazar L, Flores-Le Roux JA, Benaiges D, et al. Trends in prevalence of gestational diabetes and perinatal outcomes in Catalonia, Spain, 2006 to 2015: the Diagestcat study. Diabetes Metab Res Rev 2019;35:e3151.

14 Fadl HE, Simmons D. Trends in diabetes in pregnancy in Sweden 1998-2012. BMJ Open Diabetes Res Care 2016;4:e000221.

15 de Andrés AL, Jiménez-García R, Carrasco-Garrido P. Trends in pregestational diabetes among women delivering in Spain, 2001-2008. Int J Gynaecol Obstet 2012;117:182-3.

16 Bardenheier BH, Imperatore G, Devlin HM, et al. Trends in prepregnancy diabetes among deliveries in 19 U.S. states, 2000-2010. Am J Prev Med 2015;48:154-61.

17 Albrecht SS, Kuklina EV, Bansil P, et al. Diabetes trends among delivery hospitalizations in the U.S., 1994-2004. Diabetes Care 2010;33:768-73.

18 Coton SJ, Nazareth I, Petersen I. A cohort study of trends in the prevalence of pregestational diabetes in pregnancy recorded in UK general practice between 1995 and 2012. BMJ Open 2016;6:e009494.

19 Lascar N, Brown J, Pattison H, et al. Type 2 diabetes in adolescents and young adults. Lancet Diabetes Endocrinol 2018;6:69-80.
20 Dabelea D, Mayer-Davis EJ, Saydah S, et al. Prevalence of type 1 and type 2 diabetes among children and adolescents from 2001 to 2009. JAMA 2014;311:1778-86.

21 American Diabetes Association. 2. Classification and diagnosis of diabetes: Standards of Medical Care in Diabetes-2019. Diabetes Care 2019;42:S13-28.

22 Blumer I, Hadar E, Hadden DR, et al. Diabetes and pregnancy: an endocrine Society clinical practice guideline. J Clin Endocrinol Metab 2013;98:4227-49.

23 International Association of Diabetes and Pregnancy Study Groups Consensus Panel, Metzger BE, Gabbe SG, et al. International association of diabetes and pregnancy study groups recommendations on the diagnosis and classification of hyperglycemia in pregnancy. Diabetes Care 2010;33:e98-82.

24 American Diabetes Association. Diagnosis and classification of diabetes mellitus. Diabetes Care 2011;34(Suppl 1):S62-9.

25 Patterson CC, Gyürüs E, Rosenbauer J, et al. Trends in childhood type 1 diabetes incidence in Europe during 1989-2008: evidence of non-uniformity over time in rates of increase. Diabetologia 2012:55:2142-7.

26 Patterson CC, Harjutsalo V, Rosenbauer J, et al. Trends and cyclical variation in the incidence of childhood type 1 diabetes in 26 European centres in the 25 year period 1989-2013: a multicentre prospective registration study. Diabetologia 2019;62:408-17.

27 Murphy HR, Bell R, Cartwright C, et al. Improved pregnancy outcomes in women with type 1 and type 2 diabetes but substantial clinic-to-clinic variations: a prospective nationwide study. Diabetologia 2017;60:1668-77.

28 Balsells M, García-Patterson A, Gich I, et al. Maternal and fetal outcome in women with type 2 versus type 1 diabetes mellitus: a systematic review and metaanalysis. $J$ Clin Endocrinol Metab 2009;94:4284-91.

29 Jovanovič L, Liang Y, Weng W, et al. Trends in the incidence of diabetes, its clinical sequelae, and associated costs in pregnancy. Diabetes Metab Res Rev 2015;31:707-16.

30 Grupo Español de Diabetes y Embarazo. Asistencia a la gestante con diabetes. Guía de práctica clínica actualizada en 2014. Av en Diabetol 2015;31:45-59.

31 Vahratian A, Zhang J, Troendle JF, et al. Labor progression and risk of cesarean delivery in electively induced nulliparas. Obstet Gynecol 2005;105:698-704

32 Heffner LJ, Elkin E, Fretts RC. Impact of labor induction, gestational age, and maternal age on cesarean delivery rates. Obstet Gynecol 2003;102:287-93.

33 Wood S, Cooper S, Ross S. Does induction of labour increase the risk of caesarean section? A systematic review and meta-analysis of trials in women with intact membranes. BJOG 2014;121:674-85. discussion 685.

34 Walker KF, Bugg GJ, Macpherson M, et al. Randomized trial of labor induction in women 35 years of age or older. N Engl J Med 2016:374:813-22.

35 Kjos SL, Henry OA, Montoro M, et al. Insulin-requiring diabetes in pregnancy: a randomized trial of active induction of labor and expectant management. Am J Obstet Gynecol 1993;169:611-5.

36 Remsberg KE, McKeown RE, McFarland KF, et al. Diabetes in pregnancy and cesarean delivery. Diabetes Care 1999;22:1561-7.

37 Auger N, Luo Z-C, Nuyt AM, et al. Secular trends in preeclampsia incidence and outcomes in a large Canada database: a longitudinal study over 24 years. Can J Cardiol 2016;32:987. e15-23.

38 Wallis AB, Saftlas AF, Hsia J, et al. Secular trends in the rates of preeclampsia, eclampsia, and gestational hypertension, United States, 1987-2004. Am J Hypertens 2008;21:521-6.

39 Hutcheon JA, Lisonkova S, Joseph KS. Epidemiology of preeclampsia and the other hypertensive disorders of pregnancy. Best Pract Res Clin Obstet Gynaecol 2011;25:391-403.

40 Khalifeh A, Breathnach F, Coulter-Smith S, et al. Changing trends in diabetes mellitus in pregnancy. J Obstet Gynaecol 2014;34:135-7.

41 Grupo Español de Diabetes y Embarazo. Guía asistencial de Diabetes Mellitus y Embarazo ( $3^{\mathrm{a}}$ edición). Av en Diabetol 2006:73-87.

42 Rubio JA, Ontañón M, Perea V. Asistencia sanitaria de la mujer gestante con diabetes en España: aproximación usando un cuestionario. Endocrinología y Nutrición 2016;63:1-8. 